Medscape, Mitsubishi Tanabe Pharma, MSD, Novartis, Pfizer, Roche, Sanofi, Serodapharm, Topadur, Target Bioscience and UCB. Patent issued "mir-29 for the treatment of systemic sclerosis" (US8247389, EP2331143)., Consultant of: has/had consultancy relationship and/or has received research funding in the area of potential treatments for systemic sclerosis and its complications from (last three years): Abbvie, Acceleron Pharma, Amgen, AnaMar, Arxx Therapeutics, Baecon Discovery, Blade Therapeutics, Bayer, Boehringer Ingelheim, ChemomAb, Corbus Pharmaceuticals, CSL Behring, Galapagos NV, Glenmark Pharmaceuticals, GSK, Horizon (Curzion) Pharmaceuticals, Inventiva, iQvia, Italfarmaco, iQone, Kymera Therapeutics, Lilly, Medac, Medscape, Mitsubishi Tanabe Pharma, MSD, Novartis, Pfizer, Roche, Sanofi, Serodapharm, Topadur, Target Bioscience and UCB. Patent issued "mir-29 for the treatment of systemic sclerosis" (US8247389, EP2331143)., Grant/research support from: Kymera Therapeutics, Mitsubishi Tanabe, Armando Gabrielli: None declared, Stefan Heitmann: None declared, Nicolas Hunzelmann: None declared, Carlomaurizio Montecucco: None declared, Jadranka Morovic-Vergles: None declared, Camillo Ribi: None declared, Andrea Doria: None declared, Yannick Allanore: None declared DOI: 10.1136/annrheumdis-2021-eular.3048

\section{POS0319 PERFORMANCE OF PATIENT REPORTED OUTCOMES (PROS) IN SCLERODERMA PATIENTS WITH REDUCED LUNG FUNCTION IN AN OBSERVATIONAL COHORT}

M. G. Lazzaroni ${ }^{1}$, G. Abignano ${ }^{2,3}$, M. Wilson ${ }^{2}$, V. Kakkar ${ }^{2}$, F. Del Galdo ${ }^{2} .{ }^{1} A S S T$ Spedali Civili of Brescia, University of Brescia, Rheumatology and Clinical Immunology, Brescia, Italy; ${ }^{2}$ University of Leeds, Institute of Rheumatic and Musculoskeletal Medicine and NIHR Biomedical Research Centre, Leeds, United Kingdom; ${ }^{3}$ Azienda Ospedaliera San Carlo, Rheumatology, Potenza, Italy

Background: Patient Reported Outcomes (PROs) are used to capture disease impact on patients' Health Related Quality of Life (HRQoL) and they have been increasingly used as endpoints in clinical trials for Systemic Sclerosis (SSc). The DeSScipher project within the EUSTAR group highlighted dyspnea as one of the factors more strongly related with the highest SHAQ scores (1). Nevertheless, the SENSCIS trial in SSc-ILD (2) showed the efficacy of Nintedanib in reducing the annual rate of FVC loss, as compared to placebo, without significant changes in the PROs used as secondary endpoints (St. George's Respiratory Questionnaire and the FACIT-Dyspnoea questionnaire). Since patient's perspective is a crucial determinant to define the overall relevance of an intervention, the performance of PROs in reflecting different lung functional stages is a relevant issue in SSc. Objectives: To analyse in a prospective SSc cohort the inference of reduced lung function as measured by FVC on median PRO scores and the correlation among distinct commonly used PROs.

Methods: A cross-sectional study was performed on data exported from the STRIKE database regarding SSc patients followed in Leeds Scleroderma Programme for SSc. Data included records of periodical visits with scores of different PROs commonly used in SSc (Scleroderma-Health Assessment Questionnaire (SHAQ), Cochin Hand Function Scale (CHFS) and Borg dyspnoea scale) and FVC\%-predicted (\%pFVC) and DLCO\%-predicted (\%pDLCO). SHAQ score was calculated as the mean value of $\mathrm{HAQ}(0-3)$ with the average of the 7-VAS scores divided by 3.33. The 7-VAS (score 1-10) were 1: pain, 2: general function, 3: arthritis, 4: gastro-intestinal, 5: dyspnoea, 6: Raynaud Phenomena; 7: digital ulcers. The correlation of FVC with distinct PROs, and the inter-PRO correlation, were analysed through the non-parametric Spearman test.

Results: Complete data were available from 182 visits of 87 SSc patients (41 with diffuse and 46 with limited cutaneous involvement). Mean \%pFVC was 95.16 \pm 24.93 (median 95) and mean \%pDLCO was 59.31 \pm 16.51 (median 59). Overall, FVC and DLCO showed a moderate correlation with SHAQ $(r=-0.36, p<0.001$ and $r=-0.24, p: 0.001$ respectively), while Borg score showed a stronger negative correlation with FVC and DLCO ( $\mathrm{r}:-0.42$ and $\mathrm{r}-0.38, \mathrm{p}<0.001$ for both). In a sub-analysis of patients grouped by FVC, patients with FVC $50-70 \%$ showed a significant correlation of FVC with SHAQ ( $r=-0.47, p: 0.012)$, which was not present in patients with FVC 70-90\% (r:-0.23, p:0.13). VAS-5 dyspnoea and Borg were not associated with FVC in these two subgroups of patients.

Inter PROs analysis showed that CHFS score had a stronger correlation with $\mathrm{SHAQ}$ than Borg dyspnoea score in the overall population ( $r: 0.86 \mathrm{vs}$. $r: 0.57$, both $\mathrm{p}<0.001)$.

Conclusion: The analysis of a single centre prospective cohort of SSc patients, suggests a small inference of lung function in the overall SHAQ. The stronger correlation of SHAQ with CHFS, than with Borg score, suggests a higher weight of hand function on SHAQ in this population with relatively conserved lung function. In patients with $\% p F V C<70 \%$, the correlation with SHAQ was stronger than in patients with \%pFVC $>70 \%$, suggesting that mild reductions in FVC might not be perceived by the patients, or at least they might not modify HrQoL as measured by SHAQ.

REFERENCES:

[1] Jaeger VK, et al. Rheumatology 2017;57(3):441-50.
[2] Distler O, et al. N Engl J Med 2019; 380:2518-2528.

Disclosure of Interests: Maria Grazia Lazzaroni Grant/research support from: Boehringer-Ingelheim, Giuseppina Abignano: None declared, Michelle Wilson: None declared, Vishal Kakkar: None declared, Francesco Del Galdo Consultant of: Astra-Zeneca, Boehringer-Ingelheim, Mitsubishi-Tanabe, Capella Biosciences, Kymab, Actelion, Grant/research support from: Astra-Zeneca, Boehringer-Ingelheim, Mitsubishi-Tanabe, Capella Biosciences, Kymab, Actelion DOI: 10.1136/annrheumdis-2021-eular.3505

\section{POS0320 POOR PROGNOSIS PREDICTION IN ANTI-MDA5 POSITIVE DERMATOMYOSITIS ASSOCIATED WITH INTERSTITIAL LUNG DISEASE: THE CROSS-CAR DECISION TREE MODEL}

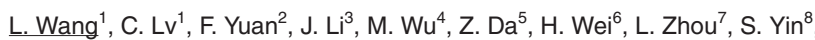
J. Wu ${ }^{9}$, W. Tan ${ }^{1} .{ }^{1}$ The First Affiliated Hospital of Nanjing Medical University, Department of Rheumatology, Nanjing, China; ${ }^{2}$ The Affiliated Wuxi People's Hospital of Nanjing Medical University, Department of Rheumatology, Wuxi, China; ${ }^{3}$ Huai'an First People's Hospital, Department of Rheumatology, Huai'an, China; ${ }^{4}$ The Third Affiliated Hospital of Soochow University, Department of Rheumatology, Changzhou, China; ${ }^{5}$ Affiliated Hospital of Nantong University, Department of Rheumatology, Nantong, China; ${ }^{6}$ Northern Jiangsu People's Hospital, Department of Rheumatology, Yangzhou, China; ${ }^{7}$ Changzhou Second People's Hospital Affiliated to Nanjing Medical University, Department of Rheumatology, Changzhou, China; ${ }^{8}$ Affiliated Hospital of Xuzhou Medical University, Department of Rheumatology, Xuzhou, China; ${ }^{9}$ The First Affiliated Hospital of Soochow University, Department of Rheumatology, Suzhou, China

Background: The prognosis of anti-melanoma differentiation-associated gene 5 positive dermatomyositis (anti-MDA5+ DM) - associated interstitial lung disease (ILD) is poor and heterogeneity.

Objectives: The aim of this study was to evaluate prognostic factors and to develop a simple and generally applicable bedside decision tree model for predicting outcomes in patients with anti-MDA5+ DM and to guide treatment.

Methods: We analyzed data for 246 anti-MDA5+ DM patients from Myositis Study Group-Jiangsu, a multicenter cohort across eighteen tertiary hospitals in Jiangsu province, from March 2019 to October 2020. The primary end point was all-cause death, and the secondary end point was occurring of rapidly progressive-ILD (rp-ILD). We used a multivariable Cox proportional hazards model to identify the independent prognostic risk factors of death and rp-ILD respectively. A decision-tree prediction model was developed by using data from 10 hospital of southern region $(n=163)$, with validation by using contemporaneous data from northern region $(\mathrm{n}=83)$.

Results: To assess the risk of rp-ILD, we developed a combined risk score, the CROSS score, that included the following values and scores: C-reactive protein $(\leq 8 \mathrm{mg} / \mathrm{L}, 0 ;>8 \mathrm{mg} / \mathrm{L}, 3)$, anti-Ro52 antibody (negative, 0; positive, 4), Sex (Female, 0; Male, 2) and Short course of disease (More than 3 months, 0; Less than 3 months, 2). The mortality risk was identified by the CAR score, including C-reactive protein $(\leq 8 \mathrm{mg} / \mathrm{L}, 0 ;>8 \mathrm{mg} / \mathrm{L}, 1)$, Alanine Transaminase ( $\leq 50 \mathrm{units} / \mathrm{L}, 0$; $>50$ units/L, 1) and rp-ILD (non-rpILD, 0; rp-ILD, 3). We divided patients into three risk groups according to the CROSS score: low, 0 to 3; medium, 4 to 7; and high 8-11. And then Use of a simple decision tree prediction model permitted strat ification into three different outcome prediction groups. High-risk patients had significantly higher mortality rates than low- and medium-risk patients in both discovery and validation cohorts $(p<0.0001)$.

Conclusion: The CROSS-CAR decision tree model is easy to evaluate the poor prognostic risk in MDA5+ DM patients during any follow-up period. Unnecessary lung examination, such as chest CT scan and arterial blood gas analysis was avoided in low- and medium- rpILD risk patients. The special ambulance, with red cross sign tagged on car in China, may help to screen the high risk patients and to guide further treatment.

Disclosure of Interests: None declared

DOI: 10.1136/annrheumdis-2021-eular.3514

\section{POS0321 \\ USE OF HYDROXYCHLOROQUINE AND SYSTEMIC SCLEROSIS: RESULTS FROM A PROSPECTIVE OBSERVATIONAL STUDY ON THE EUSTAR COHORT}

S. Bellando Randone ${ }^{1}$, H. Wilhalme ${ }^{2}$, C. Bruni ${ }^{1}$, E. Siegert ${ }^{3}$, P. Airo ${ }^{4}$, R. Irace ${ }^{5}$ O. Distler ${ }^{6}$, A. Doria ${ }^{7}$, L. P. Ananieva ${ }^{8}$, L. Czirják ${ }^{9}$, C. Denton ${ }^{10}$, Y. Allanore ${ }^{11}$, V. Riccieri ${ }^{12}$, A. Vacca $^{13}$, I. Foeldvari ${ }^{14}$, A. M. Hoffmann-Vold ${ }^{15}$, A. Gabrielli $i^{16}$, M. Matucci-Cerinic ${ }^{1}$, D. Furst ${ }^{1,2}$ on behalf of EUSTAR. ${ }^{1}$ AOU Careggi University of Florence, Division of Rheumatology, Florence, Italy; ${ }^{2}$ University of California at Los Angeles, Department of Medicine, Division of Rheumatology, Los Angeles, United States of America; ${ }^{3}$ Charité University Hospital, Rheumatology, Berlin, Germany; ${ }^{4}$ Spedali Civili di Brescia, Servizio di Reumatologia Allergologia e Immunologia Clinica, Brescia, Italy; ${ }^{5}$ II University of Naples, 
Division of Rheumatology, Naples, Italy; ${ }^{6}$ University Hospital Zurich, University of Zurich, Department of Rheumatology, Zurich, Switzerland; ${ }^{7}$ University of Padova, Rheumatology Unit, Department of Medicine (DIMED), Padova, Italy; ${ }^{8}$ VA Nasonova Institute of Rheumatology, Laboratory of Microcirculation and Inflammation, Moscow, Russian Federation; ${ }^{9}$ University of Pecs, Department of Rheumatology and Immunology, Pecs, Hungary; ${ }^{10}$ University College London, Royal Free Hospital, Department of Rheumatology, London, United Kingdom: ${ }^{11}$ Hopital Cochin Paris, Division of Rheumatology, Paris, France; 12" La Sapienza" University, 11Department of Internal Medicine and Clinical Specialties, Rheumatology Unit, Rome, Italy; ${ }^{13}$ University of Cagliari-Policlinico Universitario, II Chair of Rheumatology, Monserrato, Italy; ${ }^{14} \mathrm{Hamburg}$ Centre for Pediatric Rheumatology, Centre for Paediatric and Adolescent Rheumatology, Hamburg, Germany; ${ }^{15}$ Oslo University Hospital, Department of Rheumatology, Oslo, Norway; ${ }^{16}$ Università Politecnica delle Marche and Ospedali Riuniti, Department of Medical Science and Surgery, Section of Clinical Medicine, Ancona, Italy

Background: Hydroxychloroquine (HCQ) is a well-tolerated drug that contributes to downregulating the immune response against autoantigens and it has been used in several autoimmune diseases. In systemic sclerosis (SSc) it is used to treat inflammatory arthritis without proof of efficacy.

Objectives: Our aim was to evaluate the use of $\mathrm{HCQ}$ and its impact on Health Assessment Questionnaire disability index (HAQ-DI) and the Cochin Hand Function Status (CHFS). in a large SSc cohort compared to a propensity matched group of SSc patients not using HCQ.

Methods: SSc patients from the European Scleroderma Trials and Research (EUSTAR) data base treated with HCQ for at least 6 months were evaluated. Demographic and clinical data, concomitant drugs, duration of $\mathrm{HCQ}$ treatment and reasons for its discontinuation, HAQ-DI and CHFS (at least 2 evaluation) were recorded and were the outcome variables of interest. Statistical analysis was performed using propensity score matching for age, gender, disease duration, corticosteroids, immunosuppressives, vasoactive drugs, DMARDs in a 3:1 control:HCQ ratio. Standard descriptive statistics and Student's t-test and Chi-square test were used to assess the propensity-matched groups.

Results: 1,636 of 17,805 SSc patients $(9.2 \%)$ were treated with HCQ for at least 6 months; out of these 3\% (50/1636). had at least a baseline and follow-up HAQ-DI evaluation, (and 44/1636 (2.7\%) had at least a baseline and follow-up $\mathrm{CHFS}$ evaluation. Propensity matching assured that pts were matched for demographic variables such as gender (mean on HCQ vs no HCQ:femals:92.0 vs 85.3$)$, age(49.8 vs $49.97 \mathrm{yrs})$ disease duration(8.3 vs $9.1 \mathrm{yrs})$, limited disease $(55.3$ vs $62.6 \%)$ as well as background medications $(P>0.1-0.9)$. We did not find any significant changes in HAQ or CHFS (difference in slope) over 365 days of treatment, comparing the $\mathrm{HCQ}$-treated group to the non-HCQ treated patients $(\mathrm{p}=0.240$ for both (Figure 1$)$.

Conclusion: Results from the EUSTAR registry showed that HCQ was used by $9.2 \%$ of SSc patients. HCQ use did not improve the HAQ or CHFS, comparing $\mathrm{HCQ}$ users to non-HCQ users.

Figure 1. HAQ trend and CHFS trend over the time
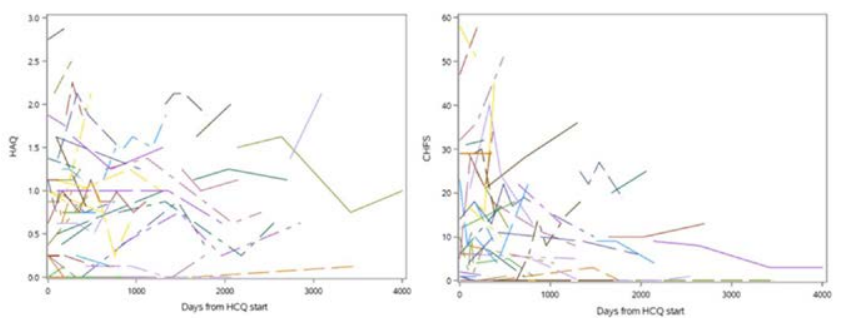

Disclosure of Interests: Silvia Bellando Randone: None declared, Holly Wilhalme: None declared, Cosimo Bruni: None declared, Elise Siegert: None declared, Paolo Airò: None declared, Rosaria Irace: None declared, Oliver Distler: None declared, Andrea Doria: None declared, Lidia P. Ananieva: None declared, László Czirják: None declared, Christopher Denton: None declared, Yannick Allanore: None declared, Valeria Riccieri: None declared, ALESSANDRA VACCA: None declared, Ivan Foeldvari Consultant of: Gilead, Novartis, Pfizer, Hexal, BMS, Sanofi, MEDAC, Anna-Maria Hoffmann-Vold Speakers bureau: Actelion, Boehringer Ingelheim, Roche, Merck Sharp \& Dohme, Lilly and Medscape, Consultant of: Actelion, Boehringer Ingelheim, Roche, Bayer, ARXX, and Medscape, Grant/research support from: Boehringer Ingelheim, Armando Gabrielli: None declared, Marco Matucci-Cerinic: None declared, Daniel Furst: None declared

DOI: 10.1136/annrheumdis-2021-eular.3725

\section{POS0322 CORRELATION BETWEEN QUANTITATIVE COMPUTED TOMOGRAPHY AND DISEASE ACTIVITY IN SYSTEMIC SCLEROSIS}

D. Sambataro ${ }^{1,2}$, M. Orlandi $^{3}$, M. Colaci ${ }^{1,1}$, L. Malatino ${ }^{1}$, G. Sambataro ${ }^{1,2}$ A. Ariani ${ }^{4} .{ }^{1}$ University of Catania, Department of Clinical and Experimental Medicine, Catania, Italy; ${ }^{2}$ Artroreuma srl, Outpatient of Rheumatology associated to Italian National Health System, Mascalucia, Italy; ${ }^{3}$ University of Florence, Department of Experimental and Clinical Medicine, Florence, Italy; ${ }^{4}$ Azienda Ospedaliero Universitaria di Parma, Department of Medicine, Parma, Italy

Background: High Resolution Computed Tomography (HRCT) is the gold standard to evaluate Interstitial Lung Diseases (ILDs) extent and severity. Quantitative Computed Tomography (QCT) is a promising tool as it provides an operator-independent assessment of ILD extent. Even if there are emerging data on QCT in Systemic Sclerosis (SSc), its correlation with disease activity (DA) has not been yet studied.

Objectives: To evaluate the correlation between QCT score and DA in an Italian cohort of SSc patients.

Methods: A multicentric, observational study was conducted in three Italian rheumatological centers.

Adult SSc patients classified according to the ACR/EULAR 2013 criteria [1] were assessed with pulmonary function tests, HRCT and for DA. CT images were analyzed quantitatively with the densitometric radiomic data obtained through a free open software - Mean lung attenuation (MLA), Standard Deviation (SD), Kurtosis, Skewness and Lung volume. DA assessment was conducted according to EUSTAR index [2]: a score $\geq 2.5$ was considered indicative of high disease activity.

Age below 18 and pregnancy were considered exclusion criteria. We used Student's T test to evaluate the means of the parameters, Pearson's $r$ test for correlations, receiver operating characteristics curve to define the cutoff values of the significant details, and linear regression with collinearity test to define the role of the details. $P$ value $<0.05$ was considered statistically significant.

Results: Sixty patients were enrolled (male 8, female 52), with mean age 53.2 years (SD 15.6) and mean disease duration 5.3 years (SD 4.2). QCT indexes distribution was different in high DA vs low DA SSc patients. In particular mean lung attenuation (MLA, -834.7 vs $-812.1, p=0.03$ ), standard deviation (95.9 vs $102, p=0.03$ ), skewness (2.2 vs $1.7, P=0.006$ ) and kurtosis ( 5.5 vs $3.3, p=0.009$ ) of the parenchymal lung and skewness ( 3.1 vs $2.8, p=0.03$ ) of the whole lung were statistically different. DA correlates with $\mathrm{MLA}(r=0.28, p=0.003)$, standard deviation $(r=0.21, p=0.02)$, skewness $(r=-0.32, p=0.001)$ and kurtosis $(r=-0.29$, $p=0.001)$ of the parenchymal lung and MLA $(r=0.25, p=0.006)$, skewness $(R=-0.27, p=0.003)$, kurtosis $(r=-0.21, P=0.02)$ of the whole lung. The skewness of the parenchymal lung was the QCT index with the best performance in identifying high DA SSc patients (cutoff value $\leq 1.85$; area under the curve 0.74 , $p=0.005$; sensitivity $79.5 \%$, specificity $68.7 \%$ accuracy $76.6 \%$ ).

Conclusion: To our knowledge, this is the first study which correlate the QCT score with DA in SSc patients. Our results suggest that QCT can identify SSc patients with high DA score. This could open a scenario of new applications as an operator-independent contribution in DA scores with a potential role in clinical practice. Further studies are needed to confirm the data and to better identify the most suitable parameters for the purpose.

\section{REFERENCES:}

[1] Van den Hoogen F, et al. 2013 classification criteria for Systemic Sclerosis: and American college of rheumatology / European league against rheumatism collaborative initiative. Ann Rheum Dis 2013:72:1747-1755.

[2] Valentini G, et al. The European Scleroderma Trials and Research group (EUSTAR) task force for the development of revised activity criteria for systemic sclerosis: derivation and validation of a preliminarily revised EUSTAR activity index. Ann Rheum Dis 2017;76:270-276.

Disclosure of Interests: None declared

DOI: 10.1136/annrheumdis-2021-eular.4261

\section{POS0323}

ANTI PM-SCL ASSOCIATED AUTO IMMUNE DISEASES: MULTICENTRIC COHORT OF 128 PATIENTS

P. Breillat ${ }^{1}$, K. Mariampillai ${ }^{2}$, P. Martins ${ }^{1}$, P. Legendre ${ }^{1}$, B. Dunogue ${ }^{1}$, J. L. Charuel ${ }^{3}$, M. Miyara ${ }^{3}$, H. Vanquaethem ${ }^{4}$, F. Ackermann ${ }^{5}$, O. Benveniste, L. Mouthon ${ }^{1}, \mathrm{H}$. Nunes ${ }^{7}$, Y. Allenbach ${ }^{6}$, Y. Uzunhan ${ }^{7} .{ }^{1}$ Assistance PubliqueHôpitaux de Paris, Hôpital Cochin, Centre de Référence pour les Maladies Auto-immunes Rares, Université de Paris, Médecine Interne, Paris, France; ${ }^{2}$ Groupe Hospitalier Pitié-Salpêtrière, Centre de Recherche en Myologie, Unité Mixte de Recherche Scientifique 974, Sorbonne Université, Institut National de la Santé et de la Recherche Médicale, Institut de Myologie, Plateforme d'essais cliniques adultes I-Motion, Paris, France; ${ }^{3}$ Assistance Publique-Hôpitaux de Paris, Groupe Hospitalier Pitié-Salpêtrière, Laboratoire Immunochimie, Paris, France; ${ }^{4}$ hôpital d'instruction des armées de Bégin, 